\title{
Echocardiographic Predictors of Adverse Outcomes in Primary Pulmonary Hypertension
}

\author{
Ronald J. Raymond, MD, ${ }^{*}$ Alan L. Hinderliter, MD,* Park W. Willis, IV, MD,* David Ralph, MD, $†$ \\ Edgar J. Caldwell, MD, $\neq$ William Williams, MD, $\neq$ Neil A. Ettinger, MD,§ Nicholas S. Hill, MD,\| \\ Warren R. Summer, MD, $\uparrow$ Bennett de Boisblanc, MD, $₫$ Todd Schwartz, MS,\# Gary Koch, PHD,\# \\ Linda M. Clayton, Pharm D, ${ }^{* *}$ Maria M. Jöbsis, BA,** James W. Crow, PhD, †† Walker Long, MD, 㧊 \\ for the Primary Pulmonary Hypertension Study Group
}

\section{Chapel Hill, Research Triangle Park and Durham, North Carolina; Seattle, Washington; Portland, Maine;} St. Louis, Missouri; Providence, Rhode Island; and New Orleans, Louisana

\begin{abstract}
OBJECTIVES The aim of this study was to evaluate the relationships between echocardiographic findings and clinical outcomes in patients with severe primary pulmonary hypertension (PPH).

BACKGROUND Primary pulmonary hypertension is associated with abnormalities of right heart structure and function that contribute to the poor prognosis of the disease. Echocardiographic abnormalities associated with PPH have been described, but the prognostic significance of these findings remains poorly characterized.

METHODS Echocardiographic studies, invasive hemodynamic measurements and 6-min walk tests were performed and outcomes prospectively followed in 81 patients with severe PPH. Subjects were participants in a 12 -week randomized trial examining the effects of prostacyclin plus conventional therapy compared with conventional therapy alone.

RESULTS During the mean follow-up period of $36.9 \pm 15.4$ months, 20 patients died and 21 patients underwent transplantation. Pericardial effusion $(\mathrm{p}=0.003)$ and indexed right atrial area $(\mathrm{p}=$ $0.005)$ were predictors of mortality. Pericardial effusion $(\mathrm{p}=0.017)$, indexed right atrial area $(p=0.012)$ and the degree of septal shift in diastole $(p=0.004)$ were predictors of a composite end point of death or transplantation. In multivariable analyses incorporating clinical, hemodynamic and echocardiographic variables, pericardial effusion and an enlarged right atrium remained predictors of adverse outcomes. Six-minute walk results, mixed venous oxygen saturation and initial treatment randomization were also independently associated with a poor prognosis.

CONCLUSIONS Pericardial effusion, right atrial enlargement and septal displacement are echocardiographic abnormalities that reflect the severity of right heart failure and predict adverse outcomes in patients with severe PPH. These characteristics may help identify patients appropriate for more intensive medical therapy or earlier transplantation. (J Am Coll Cardiol 2002;39: 1214-9) (C) 2002 by the American College of Cardiology Foundation
\end{abstract}

Primary pulmonary hypertension (PPH) is a disease characterized by increased pulmonary arterial pressures and pulmonary vascular resistance. Secondary abnormalities of right heart structure and function contribute significantly to the morbidity and mortality associated with the disease $(1,2)$. As many as two-thirds of the deaths in PPH may be attributed to right heart failure $(2,3)$. Previously reported echocardiographic findings in PPH include enlargement, hypertrophy and decreased contraction of the right ventricle, as well as associated abnormalities of interventricular

From the Departments of Medicine, *University of North Carolina, Chapel Hill, North Carolina; †University of Washington, Seattle, Washington; $¥$ Maine Medical Center, Portland, Maine; §Washington University, St. Louis, Missouri; ॥Rhode Island Hospital, Providence, Rhode Island; \Louisiana State University Medical Center, New Orleans, Louisana; \#Department of Biostatistics, University of North Carolina, Chapel Hill, North Carolina; ${ }^{* *}$ GlaxoWellcome Inc., Research Triangle Park, North Carolina; † United Therapeutics, Inc., Chapel Hill, North Carolina; and

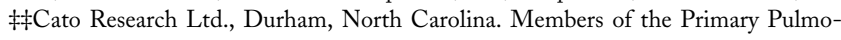
nary Hypertension Study Group are listed in the Appendix of reference 4. Supported by Glaxo Wellcome, Inc., Research Triangle Park, North Carolina.

Manuscript received August 8, 2001; revised manuscript received January 3, 2002, accepted January 16, 2002. septal motion; pericardial effusion; and tricuspid regurgitation (1,4-7). The relationship between echocardiographic variables and adverse outcomes has been described in only a few series (7-9).

We examined the associations between echocardiographic findings and clinical outcomes in patients with severe PPH who were enrolled in a trial of continuous intravenous prostacyclin. The principal results of the trial and a description of the effects of prostacyclin on the echocardiographic manifestations of the disease have been published previously $(4,10)$. We believe this to be the largest series of patients with PPH in which well-defined echocardiographic data were obtained and outcomes were prospectively followed.

\section{METHODS}

Patients. The study sample consisted of 81 patients diagnosed with PPH and New York Heart Association (NYHA) class III or IV symptoms who were enrolled in a 


$$
\begin{array}{ll}
\text { Abbreviations and Acronyms } \\
\begin{aligned}
\text { CI } & =\text { confidence interval } \\
\text { HR } & =\text { hazard ratio } \\
\text { NYHA } & =\text { New York Heart Association } \\
\text { PPH } & =\text { primary pulmonary hypertension } \\
\text { RA } & =\text { right atrium } \\
\text { RVEDA } & =\text { right ventricular end-diastolic area } \\
\text { RVESA } & =\text { right ventricular end-systolic area } \\
\text { TR } & =\text { tricuspid regurgitation }
\end{aligned}
\end{array}
$$

multicenter, randomized, open-label study examining the effects of a long-term prostacyclin infusion plus conventional therapy as compared with conventional therapy alone (10).

Study protocol. The study was approved by the institutional review committee at each participating center, and informed consent was obtained from each patient before enrollment. A detailed description of the study protocol has been reported previously (10).

Patients underwent baseline echocardiography on the first day of the trial using a predefined imaging protocol (4). All studies were recorded on VHS videocassettes and were analyzed at the core echocardiography laboratory using a digital off-line quantification system. Analyses were performed by a single echocardiographer who was blinded to the patients' treatment assignment and clinical information. For each variable, three representative beats were analyzed and the mean results calculated. Predefined echocardiographic variables that were analyzed included the following:

1. Right ventricular end-diastolic area (RVEDA) as measured in the apical four-chamber view by tracing the endocardial edges of the right ventricle and the plane of the tricuspid valve at end-diastole. The calculated area was divided by height to account for differences in body size.

2. Right ventricular systolic function as estimated by the right ventricular percent change in area. Areas of the right ventricle at end-systole (RVESA) and end-diastole were determined using the method described above, and the percent change in area calculated as: $100 \times$ (RVEDA - RVESA)/RVEDA.

3. Right atrial area as measured by planimetry in the apical four-chamber view at end-systole, corrected for height.

4. The eccentricity index, a measure of septal displacement, measured in the parasternal short-axis view at both end-systole and end-diastole. This index was calculated by the method of Ryan et al. (11) as D2/D1, where D2 is the minor-axis dimension of the left ventricle parallel to the septum and D1 is the minor-axis dimension perpendicular to and bisecting the septum.

5. Pericardial effusion, defined as a distinct diastolic separation of the pericardial layers posterior to the heart on the parasternal long-axis and short-axis views.

6. Severity of tricuspid regurgitation (TR) quantified as the ratio of the maximal regurgitant jet area and area of the right atrium (RA) (TR/RA ratio) in the apical fourchamber view. The outlines of the regurgitant signal and the right atrium were traced and the area determined by computerized planimetry.

7. Maximal instantaneous velocity of the tricuspid regurgitation signal measured using continuous wave Doppler from the cardiac apex.

Normal values for echocardiographic variables were generated by analyzing echocardiograms of 40 healthy young adults without significant cardiopulmonary disease who participated in an unrelated study.

All patients underwent right heart catheterization as part of the initial evaluation. Hemodynamic measurements included heart rate, mean arterial pressure, mean pulmonary capillary wedge pressure, mean pulmonary arterial and right atrial pressures, pulmonary vascular resistance, cardiac index and mixed venous oxygen saturation. An unencouraged 6-min walk test (12) was performed as an assessment of exercise capacity.

Patients were randomized to receive either a continuous infusion of intravenous prostacyclin in addition to conventional therapy or conventional therapy alone for 12 weeks. Conventional therapy included oxygen, vasodilators, diuretics, cardiac glycosides and anticoagulants, prescribed at the discretion of the individual investigator. At the conclusion of the 12-week protocol, all patients were offered the option of receiving chronic prostacyclin by continuous infusion as part of an open-label, observational study, and all but five patients chose this form of therapy. Follow-up data classifying each patient as alive, transplanted or dead was available for at least 12 weeks in all patients and for at least one year in 74 of the 81 patients enrolled. The primary end point examined was all-cause mortality. A composite end point of death or transplantation was also analyzed.

Data analysis. All analyses were performed using the SAS statistical software (SAS Institute, Cary, North Carolina). Data were summarized as mean \pm SD for continuous variables and number of subjects (\%) for categorical variables.

Cox proportional hazards modeling was used for univariable analyses of echocardiographic variables as predictors of death and of the composite end point of death or transplantation. Baseline clinical, hemodynamic and exercise variables of potential prognostic value, and treatment to which the patients were randomized were also evaluated. These included age, gender and NYHA functional class as clinical variables; heart rate, mean pulmonary arterial and right atrial pressures, pulmonary vascular resistance, cardiac index and mixed venous oxygen saturation as hemodynamic variables; and the distance walked in $6 \mathrm{~min}$ as the exercise variable. Hazard ratios (HRs) and their corresponding $95 \%$ confidence intervals $(95 \% \mathrm{CI})$ were produced from the parameter estimates and standard errors obtained from the proportional hazards models. Kaplan-Meier survival curves were created for the echocardiographic predictors that had 
Table 1. Clinical Features, Hemodynamic Characteristics and Exercise Capacity of the 81 Patients

\section{Age (yrs)}

$40 \pm 15$

Male gender (n [\%])

$22(27 \%)$

NYHA class IV (n [\%])

Heart rate (beats/min)

Mean arterial pressure $(\mathrm{mm} \mathrm{Hg})$

Mean PCWP $(\mathrm{mm} \mathrm{Hg})$

Mean PA pressure $(\mathrm{mm} \mathrm{Hg})$

Mean RA pressure $(\mathrm{mm} \mathrm{Hg})$

Pulmonary vascular resistance $(\mathrm{mm} \mathrm{Hg} / 1 / \mathrm{min})$

Cardiac index $(1 / \mathrm{min})$

Mixed venous $\mathrm{O}_{2}$ saturation (\%)

6-min walk $(\mathrm{ft}$ )

$21(26 \%)$

$83 \pm 15$

$89 \pm 11$

$10 \pm 4$

$60 \pm 13$

$12 \pm 7$

$16 \pm 7$

$2.1 \pm 0.8$

$61 \pm 13$

$966 \pm 413$

Data for continuous variables are presented as mean $\pm \mathrm{SD}$, and values for categorical values are presented as number (\%) of subjects with the stated attribute.

NYHA $=$ New York Heart Association; PA = pulmonary arterial; PCWP = pulmonary capillary wedge pressure; $\mathrm{RA}=$ right atrial.

statistical significance for the end points; when these predictors were continuous variables, patients with values above and below the median were compared.

For the multivariable analyses, an imputed dataset was produced with the overall mean value imputed for any missing values so that this dataset contained complete data for all 81 patients. The multivariable models included all variables in the echocardiographic, clinical, hemodynamic and exercise groupings, as well as the initial treatment randomization. Within each grouping, forward selection models were fit separately to both the imputed and nonimputed datasets to determine the predictors that entered with two-sided $p<0.15$. Predictors from each of the groupings with entry to either of the models for the imputed or nonimputed datasets were then combined as candidates for forward selection models on the imputed dataset. Finally, the resulting models were fit to the nonimputed dataset, and any nonsignificant predictors were removed.

\section{RESULTS}

Baseline demographic features, hemodynamic characteristics and 6-min walk results of the 81 patients are shown in Table 1. Echocardiographic values are noted in Table 2. Echocardiograms were of adequate technical quality for
Table 2. Baseline Echocardiographic Variables for the Study Population and Normal Controls

\begin{tabular}{lcc}
\hline & Patients & Normal Controls \\
\hline RV end-diastolic area & $21.2 \pm 5.1(\mathrm{n}=75)$ & $10.0 \pm 0.4(\mathrm{n}=35)$ \\
$\quad$ index $\left(\mathrm{cm}^{2} / \mathrm{m}\right)$ & & \\
RV \% area change (\%) & $18.6 \pm 8.1(\mathrm{n}=75)$ & $41.5 \pm 1.2(\mathrm{n}=35)$ \\
RA area index $\left(\mathrm{cm}^{2} / \mathrm{m}\right)$ & $19.9 \pm 6.6(\mathrm{n}=78)$ & $9.1 \pm 1.7(\mathrm{n}=35)$ \\
Systolic eccentricity index & $1.7 \pm 0.4(\mathrm{n}=77)$ & $1.01 \pm 0.01(\mathrm{n}=35)$ \\
Diastolic eccentricity index & $1.9 \pm 0.5(\mathrm{n}=77)$ & $1.00 \pm 0.01(\mathrm{n}=35)$ \\
Pericardial effusion (\%) & $42(\mathrm{n}=79)$ & $0(\mathrm{n}=40)$ \\
TR/RA ratio & $0.28 \pm 0.17(\mathrm{n}=78)$ & $0.04 \pm 0.05(\mathrm{n}=40)$ \\
Maximum TR & $4.2 \pm 0.6(\mathrm{n}=70)$ & Not measured \\
$\quad$ & & \\
\hline
\end{tabular}

Data for continuous variables are presented as mean $\pm \mathrm{SD}$, and values for pericardial effusion are expressed as the percent of subjects with a significant effusion.

$\mathrm{RA}=$ right atrial; $\mathrm{RV}=$ right ventricular; $\mathrm{TR}=$ tricuspid regurgitation.

quantification of right ventricular size and function, septal curvature, pericardial effusion and TR severity in at least 75 patients, and maximal tricuspid regurgitant jet velocity was measurable in 70. During the follow-up period, 20 patients died and 21 patients underwent transplantation. Survivors were followed for a mean of $36.9 \pm 15.4$ months. Mean survival in patients who died was $7.5 \pm 6.1$ months, and mean time to surgery in patients undergoing transplantation was $14.4 \pm 10.6$ months.

The HRs, CIs and significance levels of echocardiographic variables as predictors of adverse outcomes are listed in Table 3. The presence of a pericardial effusion $(\mathrm{p}=$ $0.003)$ and the right atrial area index $(p=0.005)$ were significantly associated with mortality. The relationship of the diastolic eccentricity index with mortality was of marginal statistical significance $(p=0.074)$. The diastolic eccentricity index $(p=0.004)$, right atrial area index $(p=$ $0.012)$ and presence of effusion $(p=0.017)$ were associated with the composite end point of death or transplantation. Kaplan-Meier survival curves for significant echocardiographic predictors of adverse outcomes are shown in Figure 1.

As illustrated in Table 4, proportional hazards modeling also demonstrated that death was associated with poor 6 -min walk results $(\mathrm{p}=0.001)$, high right atrial pressure $(\mathrm{p}=0.004)$, advanced NYHA functional class $(\mathrm{p}=0.005)$,

Table 3. Results of Univariable Proportional Hazards Modeling: Hazard Ratios, 95\% Confidence Intervals and $\mathrm{p}$ Values for Echocardiographic Variables

\begin{tabular}{lllllcc}
\hline & \multicolumn{2}{c}{ Death } & & \multicolumn{2}{c}{ Composite End Point } \\
\cline { 2 - 3 } & HR (95\% CI) & p Value & & HR (95\% CI) & p Value \\
\hline RV end-diastolic area index $\left(5 \mathrm{~cm}^{2} / \mathrm{m}\right)$ & $1.34(0.90-1.98)$ & 0.148 & & $1.26(0.95-1.66)$ & 0.110 \\
RV \% area change $(10 \%)$ & $0.70(0.39-1.25)$ & & 0.225 & & $0.86(0.57-1.28)$ & 0.454 \\
RA area index $\left(5 \mathrm{~cm}^{2} / \mathrm{m}\right)$ & $1.54(1.13-2.10)$ & & 0.005 & & $1.33(1.06-1.66)$ & 0.012 \\
Systolic eccentricity index $(0.5 \mathrm{U})$ & $1.41(0.94-2.13)$ & & 0.096 & & $1.20(0.86-1.68)$ & 0.286 \\
Diastolic eccentricity index $(0.5 \mathrm{U})$ & $1.39(0.97-2.00)$ & & 0.074 & & $1.45(1.12-1.86)$ & 0.004 \\
Pericardial effusion $(\mathrm{Y} / \mathrm{N})$ & $3.89(1.49-10.14)$ & & 0.003 & & $2.08(1.12-3.86)$ & 0.017 \\
TR/RA ratio $(0.2 \mathrm{U})$ & $1.24(0.75-2.06)$ & & 0.400 & & $1.37(0.96-1.97)$ & 0.080 \\
Maximum TR velocity $(0.5 \mathrm{~m} / \mathrm{s})$ & $0.90(0.62-1.31)$ & & 0.591 & & $1.00(0.77-1.30)$ & 0.981 \\
\hline
\end{tabular}

Hazard ratio and confidence interval calculations were based on changes of approximately 1 SD (indicated in parentheses) for continuous variables and for presence or absence of a pericardial effusion.

$\mathrm{CI}=$ confidence interval; $\mathrm{HR}=$ hazard ratio; $\mathrm{RA}=$ right atrial; $\mathrm{RV}=$ right ventricular; $\mathrm{TR}=$ tricuspid regurgitation. 
Right atrial size
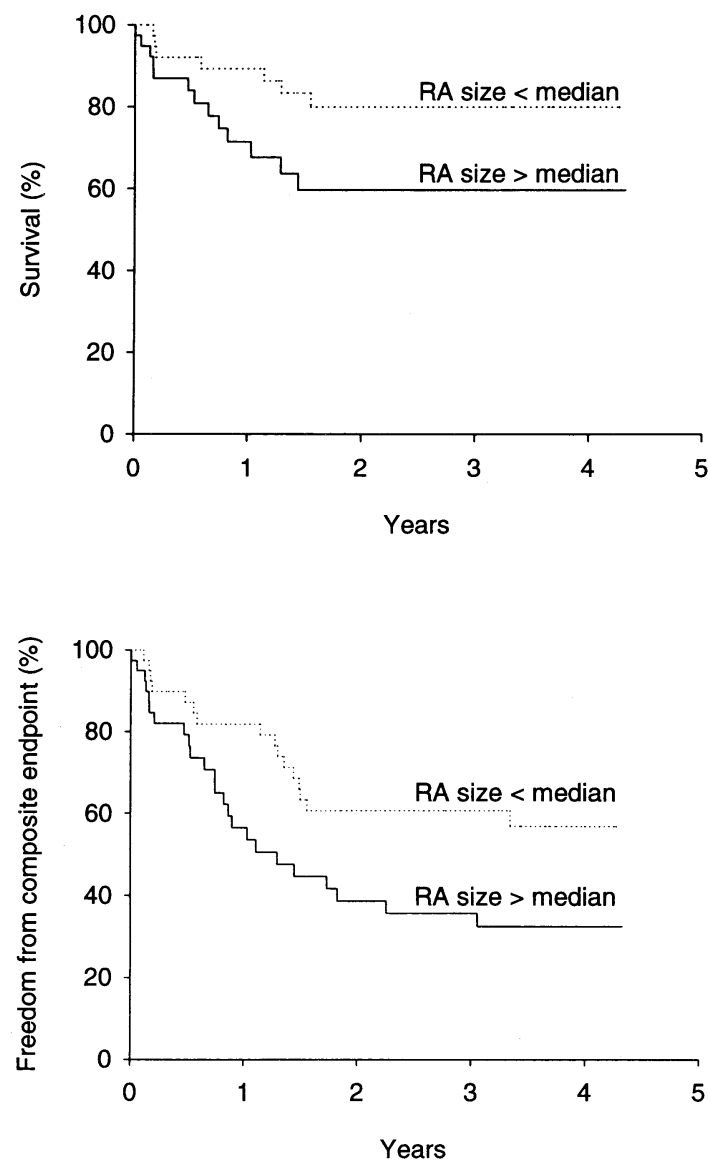

Pericardial effusion
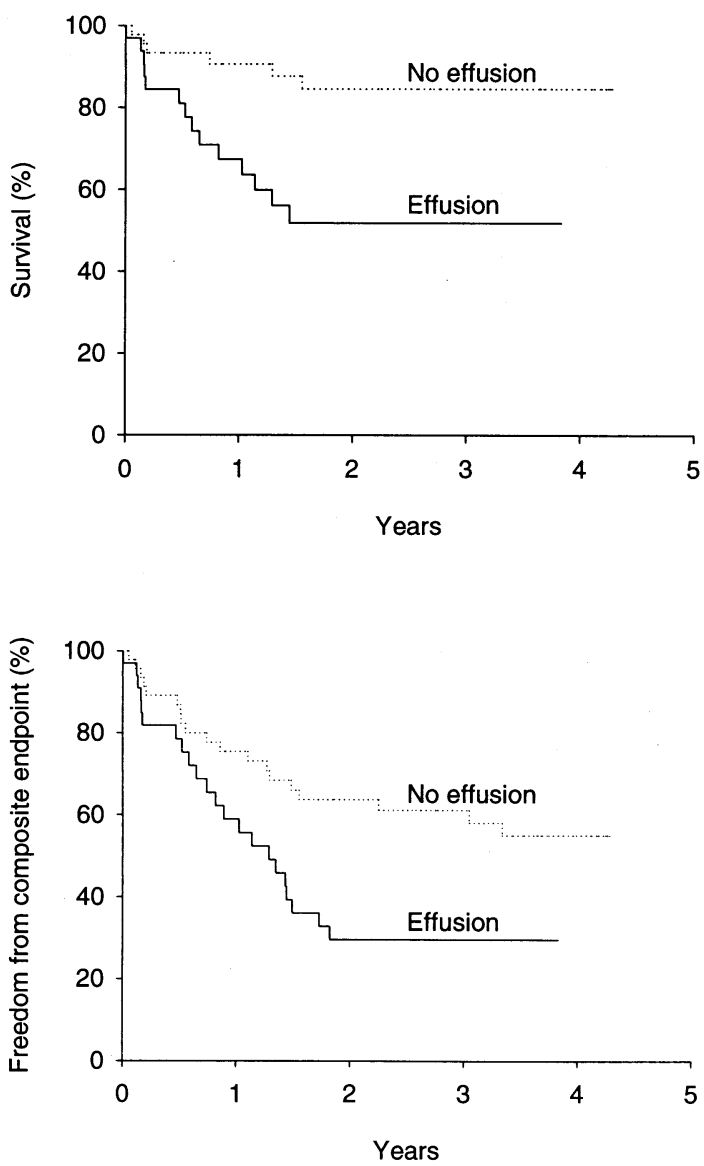

Eccentricity index
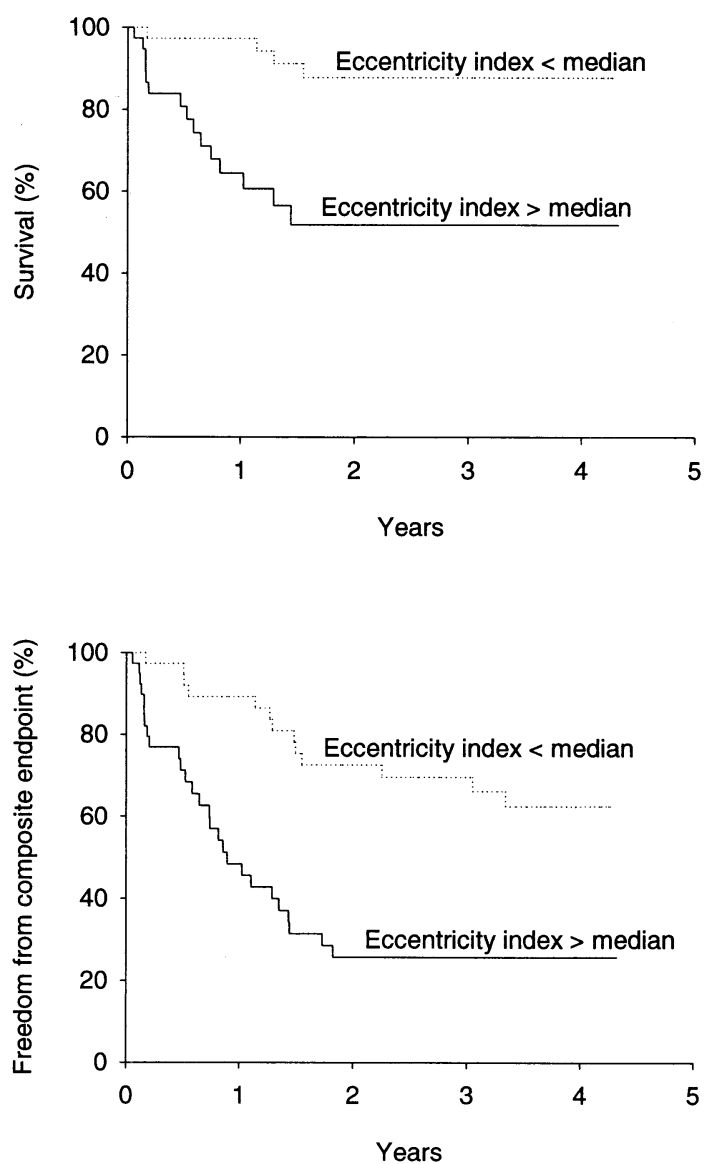

Figure 1. Kaplan-Meier survival curves for echocardiographic predictors of outcomes. By Cox proportional hazards modeling, right atrial $(\mathrm{RA})$ area index $(\mathrm{p}=0.005)$ and pericardial effusion $(\mathrm{p}=0.003)$ were significant predictors of death; the relationship of diastolic eccentricity index to mortality was of marginal statistical significance $(p=0.074)$. Right atrial area index $(p=0.012)$, pericardial effusion $(p=$ $0.017)$ and diastolic eccentricity index $(\mathrm{p}=0.004)$ were all significant predictors of a composite end point of death or transplantation. 
Table 4. Results of Univariable Proportional Hazards Modeling: Hazard Ratios, 95\% Confidence Intervals and $\mathrm{p}$ Values for Clinical and Hemodynamic Variables

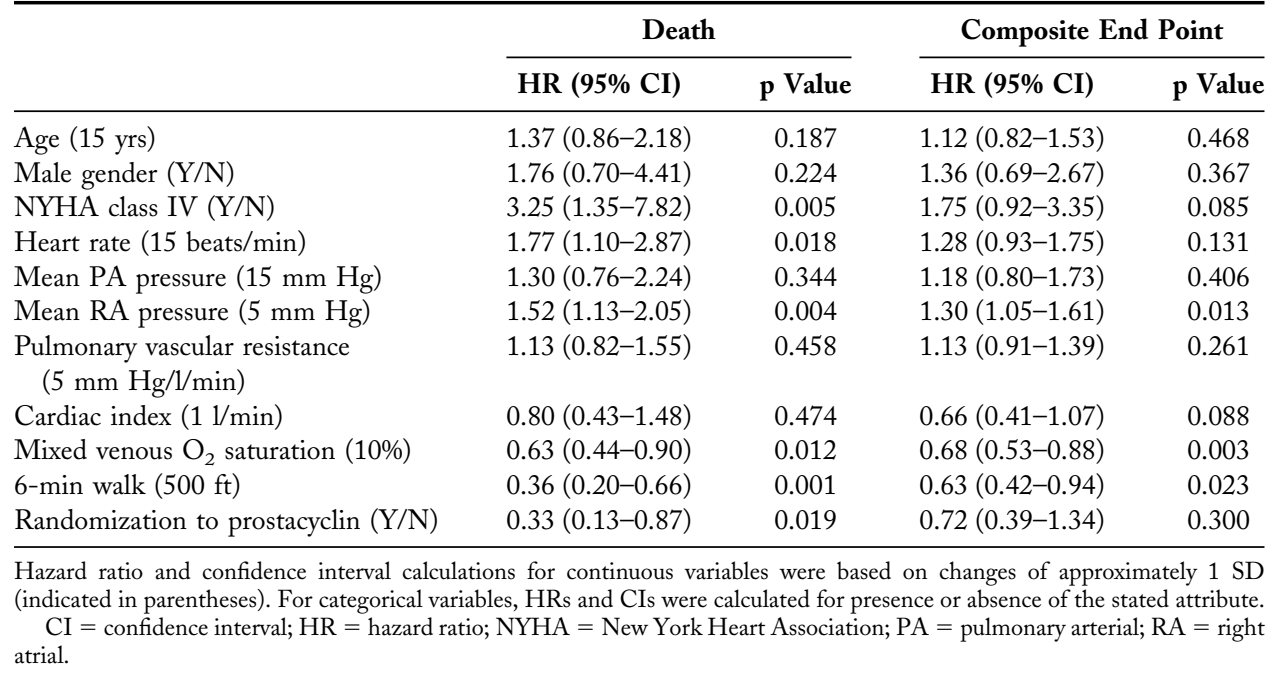

low mixed venous oxygen saturation $(p=0.012)$, high heart rate $(\mathrm{p}=0.018)$ and initial treatment randomization $(\mathrm{p}=$ 0.019). Predictors of the composite end point of death or transplantation were mixed venous oxygen saturation $(\mathrm{p}=$ $0.003)$, right atrial pressure $(\mathrm{p}=0.013)$ and distance walked in $6 \min (\mathrm{p}=0.023)$.

Results of the multivariable analysis are shown in Table 5. Pericardial effusion $(p=0.011)$ was the strongest predictor of mortality; treatment randomization $(p=0.014)$ and the distance walked in $6 \mathrm{~min}(\mathrm{p}=0.082)$ were also independent predictors. The mixed venous oxygen saturation $(\mathrm{p}=0.016)$ and indexed right atrial area $(\mathrm{p}=0.106)$ were independent predictors of the composite end point of death or transplantation.

\section{DISCUSSION}

This study identifies echocardiographic variables associated with unfavorable outcomes in the largest prospective trial of patients with PPH yet reported. The right atrial size,

Table 5. Results of Multivariable Modeling: Hazard Ratios, 95\% Confidence Intervals and p Values for Significant Multivariable Predictors of Outcome in Models That Incorporate Echocardiographic, Clinical and Hemodynamic Variables

\begin{tabular}{lll}
\hline & HR (95\% CI) & p Value \\
\hline $\begin{array}{l}\text { Significant multivariable predictors } \\
\text { of death }\end{array}$ & & \\
$\quad \begin{array}{ll}\text { Pericardial effusion }(\mathrm{Y} / \mathrm{N}) \\
\text { Randomization to prostacyclin }(\mathrm{Y} / \mathrm{N})\end{array}$ & $0.38(1.41-13.67)$ & 0.011 \\
$\quad$ 6-min walk (500 ft) & $0.56(0.29-1.08)$ & 0.014 \\
$\begin{array}{l}\text { Significant multivariable predictors } \\
\quad \text { of composite end point }\end{array}$ & 0.082 \\
$\quad$ Mixed venous $\mathrm{O}_{2}(10 \%)$ & $0.73(0.56-0.94)$ & \\
RA area index $\left(5 \mathrm{~cm}^{2} / \mathrm{m}\right)$ & $1.24(0.96-1.61)$ & 0.016 \\
\hline
\end{tabular}

Hazard ratio and confidence interval calculations were based on the changes indicated in parentheses for each continuous variable and on the presence or absence of the stated attribute for categorical variables.

$\mathrm{CI}=$ confidence interval; $\mathrm{HR}=$ hazard ratio; $\mathrm{RA}=$ right atrial. presence of a pericardial effusion and degree of septal shift were associated with mortality and a composite end point of death or transplantation. Pericardial effusion and indexed right atrial area remained significant predictors of adverse outcomes after accounting for demographic, hemodynamic and exercise variables.

Significance of the findings. Most deaths in patients with $\mathrm{PPH}$ are due to right heart failure $(2,3)$. The three echocardiographic variables most closely associated with poor outcomes in our study-right atrial area index, diastolic eccentricity index and pericardial effusion score-may all be viewed as indicators of right ventricular decompensation.

In the absence of primary abnormalities of the tricuspid valve, right atrial enlargement is generally a manifestation of high right atrial pressure due to functional tricuspid regurgitation or elevated right ventricular diastolic pressure, both consequences of right ventricular failure. In our study subjects, right atrial area index was closely correlated with right atrial pressure $(\mathrm{r}=0.72, \mathrm{p}<0.001)$ and significantly related to the TR/RA ratio $(\mathrm{r}=0.33, \mathrm{p}<0.01)$.

Pericardial effusion may also be a manifestation of right heart failure. The physiology of the formation and removal of pericardial fluid is incompletely understood. However, fluid resorption is probably via an extensive venous circulation and a system of lymphatic plexuses that are located in the subepicardial region of the heart. These ultimately drain into the RA (13). A previous analysis of our data demonstrated that effusion size is correlated with right atrial pressure (9). Thus, it is likely that the pericardial effusion observed in patients with primary pulmonary hypertension is due to impaired venous and lymphatic drainage resulting from elevated right atrial pressure.

An abnormal curvature of the interventricular septum is considered one of the echocardiographic hallmarks of pulmonary hypertension $(4,6,11,14-16)$. During diastole the septum behaves as a passive membrane between the ventricular chambers. When right ventricular diastolic pressure 
is elevated, the septum bulges toward the left ventricle. The degree of displacement correlates closely with the transeptal gradient (17) and with mean right atrial pressure (4). During systole the septal contour is influenced to some extent by contraction of the septal musculature but, in large part, still reflects the difference between right and left ventricular pressures. The eccentricity index was originally described by Ryan et al. (11) as a method of quantifying the septal shift observed in right ventricular pressure and volume overload states. An abnormal systolic eccentricity index suggests high pulmonary arterial systolic pressures. An abnormal diastolic eccentricity index, the parameter associated with adverse outcomes in our patients, implies an elevated right ventricular diastolic pressure and right ventricular failure.

Comparison with previous studies. Previous studies addressing the relationships between echocardiographic parameters and clinical outcomes in patients with $\mathrm{PPH}$ have been few in number. Eysmann et al. (7) evaluated the prognostic significance of two-dimensional echocardiographic variables in an analysis of 26 patients with $\mathrm{PPH}$ in which survivors were followed for a mean of 19.7 months. As in our study, pericardial effusion size was a significant predictor of mortality, and this variable remained significant in a multivariable analysis of echocardiographic and catheterization data.

Several previous studies have identified pulsed wave Doppler indexes of ventricular filling and right ventricular contractility that are predictors of mortality. In the analysis by Eysmann et al. (7), decreased mitral inflow $\mathrm{E} / \mathrm{A}$ ratio, shorter pulmonic acceleration time and slower tricuspid early flow deceleration were associated with poor survival. In a retrospective study of 53 patients followed at the Mayo Clinic, a Doppler-derived index combining systolic and diastolic time intervals was a significant independent predictor of adverse outcome in patients with PPH (8). Data necessary for evaluating these Doppler measures were not prospectively collected in our study.

Study limitations. The use of a composite end point of death and transplantation is subject to interinstitution variability in the criteria for and availability of transplantation. However, all centers involved were tertiary care facilities with on-site transplantation available. After the initial 12-week randomized trial period, all patients were offered prostacyclin therapy, and all but a few patients went on to receive chronic intravenous prostacyclin. Because the goal of this study was to examine the relationship between baseline echocardiographic variables and outcome, we do not believe the initial period of randomization significantly altered the results.

Conclusions. Echocardiographic variables can be identified that predict unfavorable outcomes in patients with severe PPH. These predictors reflect the severity of right heart failure and include the indexed right atrial area, pericardial effusion and degree of septal shift. An assessment of these variables may help identify patients appropriate for more intensive medical therapy or earlier transplantation. As new approaches to treatment of PPH become available and clinical trials are developed, these variables should be considered in the analysis of outcome data.

\section{Acknowledgment}

The authors gratefully acknowledge the contribution of Anne Middleton in the preparation of this manuscript.

Reprint requests and correspondence: Dr. Alan L. Hinderliter, Division of Cardiology, University of North Carolina, CB \# 7075, 338 Burnett-Womack, Chapel Hill, North Carolina 27599-7075. E-mail: hinderli@med.unc.edu.

\section{REFERENCES}

1. Rich S, Dantzker DR, Ayres SM, et al. Primary pulmonary hypertension: a national prospective study. Ann Intern Med 1987;107:216-23.

2. Fuster V, Steele PM, Edwards WD, Gersh BJ, McGoon MD, Frye RL. Primary pulmonary hypertension: natural history and the importance of thrombosis. Circulation 1984;70:580-7.

3. D'Alonzo GE, Barst RJ, Ayres SM, et al. Survival in patients with primary pulmonary hypertension: results from a national prospective registry. Ann Intern Med 1991;115:343-9.

4. Hinderliter AL, Willis PW, IV, Barst RJ, et al. Effects of long-term infusion of prostacyclin (epoprostenol) on echocardiographic measures of right ventricular structure and function in primary pulmonary hypertension. Circulation 1997;95:1479-86.

5. Rich S, Brundage BH. High-dose calcium channel-blocking therapy for primary pulmonary hypertension: evidence for long-term reduction in pulmonary arterial pressure and regression of right ventricular hypertrophy. Circulation 1987;76:135-41.

6. Goodman DJ, Harrison DC, Popp RL. Echocardiographic features of primary pulmonary hypertension. Am J Cardiol 1974;33:438-43.

7. Eysmann SB, Palevsky HI, Reichek N, Hackney K, Douglas PS Two-dimensional and Doppler-echocardiographic and cardiac catheterization correlates of survival in primary pulmonary hypertension. Circulation 1989;80:353-60.

8. Yeo TC, Dujardin KS, Tei C, Mahoney DW, McGoon MD, Seward JB. Value of a Doppler-derived index combining systolic and diastolic time intervals in predicting outcome in primary pulmonary hypertension. Am J Cardiol 1998;81:1157-61.

9. Hinderliter AL, Willis PW, IV, Long WA, et al. Pericardial effusion in patients with primary pulmonary hypertension. Am J Cardiol 1999;84:481-4.

10. Barst RJ, Rubin LJ, Long WA, et al. A comparison of continuous intravenous epoprostenol (prostacyclin) with conventional therapy for primary pulmonary hypertension. N Engl J Med 1996;334:296-301.

11. Ryan T, Petrovic O, Dillon JC, Feigenbaum H, Conley MJ, Armstrong WF. An echocardiographic index for separation of right ventricular volume and pressure overload. J Am Coll Cardiol 1985;5: 918-24.

12. Guyatt GH, Sullivan MJ, Thompson PJ. The 6-minute walk: a new measure of exercise capacity in patients with chronic heart failure. Can Med Assoc J 1985;132:919-23.

13. Miller AJ, Pick R, Johnson PJ. The production of acute pericardial effusion: the effects of varying degrees of interference with venous blood and lymph drainage from the heart muscle in the dog. Am J Cardiol 1971;28:463-6.

14. Louie EK, Rich S, Brundage BH. Doppler echocardiographic assessment of impaired left ventricular filling in patients with right ventricular pressure overload due to primary pulmonary hypertension. J Am Coll Cardiol 1986;8:1298-306.

15. Louie EK, Rich S, Levitsky S, Brundage BH. Doppler echocardiographic demonstration of the differential effects of right ventricular pressure and volume overload on left ventricular geometry and filling. J Am Coll Cardiol 1992;19:84-90.

16. Jessup M, Sutton MSJ, Weber KT, Janicki JS. The effect of chronic pulmonary hypertension on left ventricular size, function, and interventricular septal motion. Am Heart J 1987;113:1114-22.

17. Kingma I, Tyberg JV, Smith ER. Effects of diastolic transseptal pressure gradient on ventricular septal position and motion. Circulation 1983;68:1304-14. 